\title{
ÉTUDE DES GLYCOPROTÉIDES DES LAITS DE FEMME ET DE VACHE
}

\author{
J. MONTREUIL, Nicole ANTONY, J. DESCAMPS et Nicole DUQUESNE \\ Laboratoire de Chimie biologique, Faculté des Sciences, Lille.
}

Le but de nos travaux est d'étudier d'une manière comparative la composition des laits de mammifères de manière à saisir les différences qui confèrent au lait de femme ses propriétés bénéfiques pour le nourrisson et à déterminer la nature des substances qu'il conviendrait d'ajouter aux laits, autres que le lait de femme, pour réaliser leur "maternisation "). vache.

Jusqu’à présent, nos travaux ont porté uniquement sur les laits de femme et de

\section{A. Étude comparée de la physiologie de la sécrétion lactée de la femme et de la vache.}

L'étude comparative de l'évolution des glucides, des glycoprotéides et des protéines du lait de vache et du lait de femme montre qu'il existe de grandes analogies, mais aussi des différences, entre les deux types de sécrétions. Nous pouvons les résumer de la manière suivante :

$1^{\text {o }}$ Le taux du lactose augmente progressivement et atteint son maximum vers le $4^{\mathrm{e}}$ jour chez la femme, vers le $15^{\mathrm{e}}$ jour chez la vache. Le retard de la " stabilisation " du taux du lactose chez cette dernière pourrait s'expliquer par l'absence d'oligosides et de polyosides en quantité suffisante pour compenser la synthèse enzymatique du lactose, faible au début de la lactation.

$2^{\circ}$ Les laits de femme et de vache contiennent des oligosides et des polyosides : le lait de femme en renferme de 10 à $25 \mathrm{~g}$ par litre; le lait de vache n'en contient que de 1 à $2 \mathrm{~g}$ par litre. Dans les deux types de lait, le taux des osides, sauf le lactose, diminue au cours de la lactation.

$3^{0}$ L'étude pondérale et électrophorétique des caséines et des protéides du lactosérum du lait de vache montre que le lait subit, au cours de la lactation, d'importantes modifications quantitatives de ses constituants protéidiques. En particulier, les laits colostraux sont riches en protéides sériques, particulièrement en $\beta_{2}$-globulines immunes, et pauvres en protéides " lactés ". Au fur et à mesure que l'on s'éloigne du jour du vélage, le taux des globulines immunes diminue progressivement, tandis que celui de la $\beta$-lactoglobuline et de l' $\alpha$-lactalbumine augmente progressivement. Le lait de femme subit une évolution identique. 
40 L'étude des variations du taux des constituants glucidiques des glycoprotéides montre que le lait subit en outre de profondes modifications qualitatives de ses glycoprotéides. L'étude des variations des rapports oses/acides sialiques et oses/osamines est à cet égard très démonstrative.

$5^{\circ}$ Les modifications qualitatives et quantitatives des laits au cours de la lactation s'effectuent toujours d'une manière très progressive.

$6^{\circ}$ La physiologie de la sécrétion lactée présente des analogies très étroites chez la femme et chez la vache et les différences qui se manifestent sont essentiellement qualitatives.

\section{B. Etude des protéides du lait de femme et du lait de vache.}

Nous appliquons les méthodes classiques d'isolement des protéides par chromatographie sur échangeurs d'ions ou par électrophorèse préparative, non pas aux protéides totaux du lait, mais à des fractions enrichies par une méthode originale de précipitation par le sulfate d'ammonium avec gradient de concentration et gradient de $\mathrm{pH}$. On obtient de cette manière des préparations de protéides encore hétérogènes, mais enrichies en un ou plusieurs protéides, qui peuvent être caractérisés par la méthode d'immunoélectrophorèse. Nous avons appliqué ce mode opératoire à l'étude des laits de femme et de vache.

\section{I. Étude des protéides du lait de femme.}

Nos recherches ont principalement porté sur les $\beta_{2 \mathrm{~A}}$-globulines, le gynolactomucoïde I, la lactosidérophiline et les glycopeptides.

\section{$1^{\circ}$ Fractionnement des $\beta_{2 \mathrm{~A}}$-globulines.}

Le fractionnement des gynolactomucoïdes s'effectue en deux étapes : $1^{\circ}$ une première chromatographie sur des colonnes d'Amberlite XE 64 fournit deux pics obtenus par le passage successif d'une solution de citrate de sodium $0,05 \mathrm{M}$ de $\mathrm{pH} 5,2$ et d'une solution de citrate de sodium $0,50 \mathrm{M}$ de $\mathrm{pH} 6,2 ; 2^{\circ}$ un fractionnement des deux pics sur DEAEcellulose est ensuite réalisé.

a) Étude du pic $n^{\circ}$ I.

Les préparations obtenues sont très riches en glucides (environ $40 \%$ ) et en acides sialiques (environ $10 \%$ ). Elles sont hétérogènes à l'électrophorèse en gélose : deux constituants ont en effet été caractérisés, qui possèdent une grande vitesse de migration anodique.

Jusqu'à présent, toutes les tentatives d'isolement de ces composés par chromatographie sur colonnes (CM-cellulose; DEAE-cellulose; Sephadex) ont été vaines. Actuellement nous nous orientons vers les méthodes d'électrophorèse préparative sur amidon ou sur colonne de cellulose modifiée selon le procédé de Porath.

Intérêt de ces recherches. Nous avons, il y a quelques années, caractérisé dans le lait de femme un mucoïde riche en glucides que nous avons appelé " gynolactomucoïde I ". Le groupement glucidique est composé de galactose, de fucose, de $\mathrm{N}$-acétylglucosamine et d'acide sialique. Cette composition rapproche le gynolactomucoïde I des subs- 
tances de groupes sanguins. Nous tentons de l'isoler à l'état pur pour confirmer éventuellement cette hypothèse et pour étudier l'existence possible d'une activité Lactobacillus bifidus.

En effectuant ces recherches, nous venons de caractériser un nouveau mucoïde (gynolactomucoïde III) dont la nature est jusqu'à présent inconnue.

b) Étude du pic $n^{0} 2$.

Ce pic est constitué essentiellement de lactosidérophiline (gynolactomucoïde II), dont le procédé d'isolement et les propriétés physico-chimiques ont été décrites par Montreuil, Mullet et Tonnelat. Il s'agit d'un glycoprotéide spécifique du lait, qui possède la propriété de fixer réversiblement jusqu'à 6 atomes de fer par molécule et qui est différent de la sidérophiline plasmatique. Il possède une importance fondamentale dans le transport du fer de la mère à l'enfant.

Nos travaux devaient porter cette année sur une étude physico-chimique plus poussée du composé et sur l'exploration de ses propriétés biologiques. Pour mener à bien cette entreprise, de grandes quantités (plusieurs grammes) de lactosidérophiline étaient nécessaires. Bien que cette préparation ait été menée à bien, nous n'avons pu entreprendre aucune étude physico-chimique ou biologique, car nous avons constaté au cours des expériences que la lactosidérophiline était hétérogène et qu'il existait, comme dans le cas de la sidérophiline plasmatique, plusieurs types de lactosidérophiline.

\section{$2^{\circ}$ Fractionnement de la lactosidérophiline.}

Le pic no 2 (obtenu par chromatographie sur Amberlite XE 64) a été passé sur des colonnes de DEAE-cellulose dont l'élution a été réalisée par le passage successif de solutions de phosphate à des concentrations et à des $\mathrm{pH}$ variables.

On observe que plusieurs pics apparaissent, dont la nature "lactosidérophiline " a été démontrée par immunoélectrophorèse. On remarque que la position des arcs de précipitation varie d'une préparation à l'autre. Ces différences de comportement électrophorétique, associées aux différences de comportement chromatographique, impliquent qu'il existe une hétérogénéité dans la constitution de la lactosidérophiline telle qu'elle a été décrite antérieurement. Cette hétérogénéité peut être liée soit à des variations de la teneur en fer des échantillons, soit à des différences dans les propriétés physicochimiques de la partie glycoprotéidique de la molécule.

Nos recherches sont actuellement orientées vers la confirmation de l'une ou de l'autre de ces hypothèses.

\section{3o Étude des glycopeptides.}

Des fractions très riches en glucides ont été isolées. Elles sont manifestement constituées par des glycopeptides et par des polyosides de haut poids moléculaire. L'importance de ces composés, dont nous commençons l'étude, est double : 10 ils sont des produits du métabolisme intermédiaire des lactomucoïdes et des glucides libres du lait (ex-gynolactose de Polonovski et LesPagnol) et leur étude apportera des renseignements précieux sur le métabolisme encore mal connu des glycoprotéides et sur la structure de ces dernièrs; $2^{\circ}$ ils possèdent vraisemblablement une activité Lactobacillus bifidus. 


\section{II. Étude des protéides du lait de vache.}

Jusqu'à présent, nos travaux ont porté uniquement sur les globulines immunes du colostrum de vache. Nous avons en effet précisé plus haut que, dans les premiers jours de la lactation, les colostrums étaient extrêmement riches en globulines immunes et très pauvres en autres protéides.

La présence de globulines immunes dans le lait de vache est d'une importance fondamentale pour l'immunisation du veau nouveau-né. En effet, chez l'humain, les globulines immunes sont transmises à la fois par le lait et par la voie diaplacentaire grâce à la minceur du barrage placentaire. Au contraire, chez les animaux comme la truie, la jument, la vache et la chèvre, sept épaisseurs de couches cellulaires séparent la circulation fœtale et maternelle et gênent le passage des anticorps. La transmission des anticorps ne peut donc s'effectuer que par le colostrum.

A l'intérêt biologique de cette étude s'ajoute l'intérêt biochimique. En effet, on connaît encore très mal les propriétés physico-chimiques des globulines immunes du plasma à cause des difficultés d'isolement que nous avons déjà signalées. Le colostrum est pratiquement une solution pure de ces globulines immunes et représente le matériau de choix pour l'étude de ces substances, à la condition que les globulines immunes du lait soient identiques aux globulines immunes du sang. Nous nous attachons à vérifier cette dernière condition en associant à nos recherches sur les globulines immunes du lait de vache des recherches sur les globulines immunes du sérum de vache.

Le fractionnement de colostrums par le sulfate d'ammonium nous a fourni une série de précipités $\left(\mathrm{P}_{\mathbf{1}}\right.$ à $\left.\mathrm{P}_{9}\right)$ dont nous avons entrepris l'étude.

a) Étude du précipité $P_{1}$.

Nous avons obtenu une préparation de $\gamma$-globulines pures avec le précipité $\mathbf{P}_{\mathbf{1}}$ d'un colostrum. L'ultracentrifugation révèle l'existence d'un seul pic de constante de sédimenation $\mathrm{S}_{20 w}=6,5( \pm 0,2)$. La détermination du poids moléculaire donne à ce dernier une valeur de 132600 ( \pm 5500 ).

Le dosage des glucides fournit les résultats suivants :

$$
\begin{aligned}
\text { oses } & : 1,6 \% \\
\text { acides sialiques }: & 0,65 \%
\end{aligned}
$$

Actuellement, des expériences de fractionnement sur des colonnes de DEAEet de CM-cellulose sont en cours, qui démontrent l'hétérogénéité de la préparation.

\section{b) Étude du précipité $P_{4}$.}

Nous nous sommes attachés à l'étude de ce précipité parce qu'il est l'un des plus abondants et l'un des moins complexes du point de vue de la composition et parce qu'il renferme un taux très élevé de globulines immunes.

Nous avons effectué le fractionnement de cette préparation sur des colonnes de DEAE-cellulose avec une élution par gradient de concentration en phosphates et à $\mathrm{pH} 8$.

Nous avons, de cette manière, obtenu une douzaine de pics dont la présence démontre la grande hétérogénéité des globulines immunes. Nous avons limité notre étude aux pics " majeurs" à cause des quantités élevées de substance que nécessitent l'immunoélectrophorèse, l'ultracentrifugation et les différents dosages. 
๙ L'étude immunoélectrophorétique montre :

- que les différentes fractions obtenues sont de nature $\gamma$ ou $\beta_{2}$-globuliniques;

- que l'hétérogénéité chromatographique s'accompagne de différences très nettes dans le comportement électrophorétique au fur et à mesure que la concentration en phosphates augmente : les composés élués migrent plus rapidement vers l'anode;

- que les préparations obtenues sont encore hétérogènes puisque de nombreux arcs présentent des bifidités.

\section{$\beta$ Ultracentrifugation.}

Cinq fractions ont été soumises à l'ultracentrifugation. Les diagrammes confirment l'hétérogénéité de ces préparations qui, cependant, possèdent un constituant " majeur » de $S_{20 w}=6,5$ représentant de 70 à $90 \%$ de chaque préparation.

On remarque en outre :

- que les constantes de sédimentation des anticorps du lait sont aussi celles de constituants $\gamma$ ou $\beta_{2}$-globuliniques du plasma;

- que les constituants $7 \mathrm{~S}$ sont, - comme dans le plasma -, prédominants;

- que, toutefois, seules les molécules d'anticorps de $S_{20 \mathrm{w}}$ relativement faibles sont capables de traverser le "filtre mammaire": en effet, on ne trouve pas dans le lait les constituants de $\mathrm{S}_{20 w}$ élevées : $16 \mathrm{~S}, 18 \mathrm{~S}, 19 \mathrm{~S}, 28 \mathrm{~S}$ et $44 \mathrm{~S}$; ce résultat confirme les données de l'électrophorèse : seule une portion des globulines immunes passe du plasma sanguin dans le lait.

$\gamma$ Dosage des glucides.

Les dosages des glucides (oses, osamines et acides sialiques) ont été systématiquement effectués sur tous les pics obtenus par les fractionnements des précipités $\mathrm{P}_{4}$ sur des colonnes de DEAE-cellulose.

Nous résumerons les résultats de la manière suivante :

- les préparations montrent des différences très significatives dans leur teneur en glucides, qui varie de 1,67 à $2,84 \%$ d'oses et de 0,52 à $0,71 \%$ d'acides sialiques, avec des rapports oses/acides sialiques de 3,03 à 5,49 ; ce résultat confirme l'hétérogénéité des globulines immunes.

- l'analyse chromatographique des hydrolysats de globulines immunes montre que les oses constituants sont le galactose, le mannose et le fucose, dans les rapports molaires $6: 6: 3$. Les globulines immunes sont donc particulièrement riches en fucose. 